\title{
DICCIONARIO BILINGÜE LENGUA DE SEÑAS CHILENA/ESPAÑOL: UN DESAFÎO LEXICOGRÁFICO
}

\section{BILINGUAL DICTIONARY CHILEAN SIGN LANGUAGE/SPANISH: A LEXICOGRAPHY CHALLENGER}

\section{DORA ADAMO QUINTELA}

UMCE, Universidad Metropolitana de Ciencias de la Educación. Santiago, Chile dora.adamo@umce.cl

\section{XIMENA ACUÑA ROBERTSON}

UMCE, Universidad Metropolitana de Ciencias de la Educación. Santiago, Chile ximena.acuna@umce.cl

\section{IRENE CABRERA RAMÍREZ}

UMCE, Universidad Metropolitana de Ciencias de la Educación. Santiago, Chile irene.cabrera@umce.cl

\section{RESUMEN}

En este artículo se presenta el trabajo que fue desarrollado para la elaboración del Diccionario bilingüe Lengua de Señas Chilena/Español. Se da cuenta de las etapas de este proceso y de los desafíos lexicográficos que se fueron presentando. Parte de estos desafíos se debieron a la naturaleza gestual visual de la lengua de señas, cuyos signos lingüísticos ocupan en su realización formas de las manos en movimiento, en el espacio tridimensional. En el transcurso de su elaboración, participaron con distintos roles personas sordas pertenecientes a la Comunidad Sorda, quienes validaron las señas que fueron consignadas en fotografías digitales. En este diccionario se registran unidades léxicas de la Lengua de Señas Chilena (LSCh), lengua que es usada por la Comunidad Sorda de nuestro país, principalmente en la Región Metropolitana. Ésta es una lengua ágrafa que sólo se transmite a través de la comunicación cara a cara. Además, se entrega información sobre la elaboración de diccionarios en general y los distintos tipos en que se pueden organizar y, en particular, de los que registran lenguas de señas. La LSCh es el valor cultural más importante de la Comunidad Sorda de Chile, por lo que un diccionario de esta naturaleza puede contribuir a su divulgación y validación como lengua natural.

Palabras clave: Lexicografía, diccionarios, lengua de señas. 


\section{ABSTRACT}

This article presents the work that was carried out to develop the Chilean Sign Languagel Spanish bilingual dictionary. The stages of this process are described, as well as the lexicographical challenges that were faced during its development. Some of these challenges were due to the gestural and visual nature of sign language, in which linguistic signs are made of handshapes in movement, using the tridimensional space. Through the course of its elaboration deaf persons belonging to the Chilean Deaf Community participated with different roles, which gave validity to the signs that were recorded on digital photography. This dictionary contains the lexical units that belong to the sign language used in our country, as a means of communication, mainly by members of the Deaf Community in the Metropolitan Region. This language has no written system; therefore it is transmitted only through face to face communication. In addition, general information is provided about the development of dictionaries and the different ways in which they can be arranged, and in particular, those that register sign languages. Chilean Sign Language is the most important cultural value of the Deaf Community in Chile; therefore a dictionary of this nature can contribute to its dissemination and validation as a natural language.

Keywords: Lexicography, dictionaries, sign language.

Recibido: 25/06/2013. Aceptado: 19/08/2013.

\section{INTRODUCCIÓN}

$\mathrm{E}$ l trabajo que aquí se presenta es el resultado de una línea de investigación que se ha desarrollado durante los últimos veinte años y abarca varios proyectos orientados al estudio del sistema lingüístico de la Lengua de Señas Chilena (LSCh) ${ }^{1}$. Esta es la lengua natural y propia de las personas sordas que son miembros de la Comunidad Sorda de Chile (Adamo y Cabrera, 1989). Esta lengua por mucho tiempo no ha sido reconocida en nuestro país. En su desprestigio, desde los años 60, ha influido fuertemente la orientación oralista -tendencia arraigada en toda América y gran parte de Europa- que prevalece aún en la educación de las personas sordas. Esta orientación se centra en la enseñanza de la lengua oral para su emisión y la lectura labiofacial para la recepción de los mensajes.

Determinante de este enfoque educativo ha sido la decisión tomada en el Congreso de Milán de 1880, donde se declara, con argumentos esgrimidos por profesores oyentes y sin la participación de personas sordas, que lo óptimo para la edu-

${ }^{1}$ DIUMCE ED 01-1987 de la Universidad Metropolitana de Ciencias de la Educación (UMCE) y Fondecyt N N $^{\circ} 999$ /1988-1989 "Codificación de la lengua de señas chilena"; DIUMCE/FIBAS 23/05 2005-2007 "Recopilación y descripción lexicográfica para la elaboración de un diccionario bilingüe de lengua de señas chilena y español". 
cación de éstas debe ser el enfoque oralista, en el que las señas no son permitidas. Además, por muchos años, la comunicación por señas no fue considerada como la manifestación de un sistema lingüístico y concebida, erróneamente, sólo como una forma de comunicación gestual usada entre las personas sordas.

Actualmente, a lo largo del país, algunas escuelas se van encaminando paulatinamente hacia un bilingüismo, es decir, hacia una educación que considere la LSCh como la lengua natural de las personas sordas y el aprendizaje del español como segunda lengua. Una de las mayores dificultades para implementar este enfoque educativo es el poco conocimiento que los profesores tienen de la LSCh. Aun cuando se tenga el convencimiento de que esta orientación sea la mejor opción para los niños, niñas y jóvenes sordos, esto es un obstáculo importante ya que en este bilingüismo se contempla el dominio de la LSCh de modo que pueda ser usada como lengua de instrucción.

El hecho de que la lengua de seña no posea, hasta ahora, una forma práctica de ser escrita, dificulta su registro. En atención a la necesidad de consignar las señas, es que Barrientos y Tenorio (1987), profesoras de la Escuela Anglicana de Niños Sordos de Temuco, han elaborado y publicado un Manual de las señas compartidas por la Comunidad Sorda de esa ciudad.

En general, en las escuelas de niños sordos se presenta una situación particular de bilingüismo, donde la LSCh es usada principalmente entre ellos, a veces en el aula y casi siempre fuera de la sala de clases. Esto ocurre al existir el enfoque oralista en el que los procesos de enseñanza y aprendizaje se llevan a cabo por medio de la lengua oral. De este modo, los estudiantes son entrenados para la comunicación a través de la terapia del habla, para expresarse y la lectura labial, para recibir los mensajes. Aun cuando la lengua de señas en estas escuelas no es usada por los docentes ni se permite su uso en el aula, de todos modos ésta aparece en la interacción entre las y los estudiantes sordos en los recreos y fuera de la escuela.

Un estudio pionero en Chile, correspondiente a la recopilación de señas usadas por los alumnos de una escuela de sordos de Santiago, con internado, ha sido realizado por estudiantes del Departamento de Educación Diferencial de la Universidad de Chile (León, Merino y Villarroel, 1978), en el desarrollo de su trabajo de Memoria de Título. Asimismo, al inicio del nuevo milenio ha sido publicado un Manual de Señas destinado a servir de apoyo a los estudiantes del curso de LSCh que se imparte en las carreras del Departamento de Educación Diferencial de la UMCE (Lattapiat y Marín, 2000).

\subsection{Comunidad Sorda: Una minoría lingüística}

La concepción "tradicional" de persona sorda, según la perspectiva clínica o médica, la considera como una persona discapacitada, enferma, que necesita ser rehabi- 
litada a través de la enseñanza de la lengua oral, la lectura de los labios y la terapia auditiva, para así poder integrarse a la sociedad en la cual vive. En esta concepción la lengua de señas se interpone e interfiere (según argumento oralista) en la adquisición de la lengua oral.

En cambio:

- Desde una perspectiva socioantropológica se concibe a la persona sorda como perteneciente a una comunidad con una cultura, valores y lengua propios.

- Desde una perspectiva psicolingüistica se entiende la lengua de señas como la lengua natural de las personas sordas, en la que se presentan completas las propiedades de toda lengua humana.

Históricamente las personas sordas se han agrupado y organizado, formando comunidades con las mismas características de toda comunidad por compartir y tener entre ellas experiencias de vida comunes, ligadas a la educación y a la comunicación (Adamo y Cabrera, 1989). Aun cuando esta comunidad se encuentra aislada tanto lingüística como culturalmente de la sociedad oyente está integrada económicamente, como en todas las sociedades industrializadas del mundo (Massone y Johnson, 1990).

La Comunidad Sorda chilena se distingue por tener una historia y una cultura que les son propias. Sus miembros se definen como personas sordas, sin importar el grado de pérdida auditiva, también se incluyen personas oyentes vinculadas a ellos; se caracterizan principalmente por reconocerse y aceptarse a sí mismos como personas sordas. En estas agrupaciones se determinan sus metas y objetivos comunes. Su cultura se reconoce predominantemente visual, se refleja en su lengua natural como símbolo de identidad y en sus conductas sociales y valores propios. La LSCh se constituye así en uno de sus valores más importantes, como patrimonio cultural de la Comunidad Sorda de nuestro país. Es así que esta lengua se establece como su medio natural de comunicación, el cual se compone de un sistema lingüístico, con una gramática propia de modalidad visual y con el que las personas sordas construyen su identidad y se identifican como miembros de esa Comunidad (Adamo y Cabrera, 1989).

Los estudios que se han realizado para describir la gramática de la LSCh (Acuña, 1997; Acuña, Adamo, Cabrera y Lattapiat, 1999; Adamo, Cabrera, Lattapiat y Acuña, 1999; Adamo, Acuña, Cabrera y Lattapiat, 2003); conocer su uso y desarrollo a través de la narrativa infantil (Acuña, Adamo, Cabrera y Lissi, 2012) y hacer aproximaciones a la visión de mundo de las personas sordas² (Acuña, Adamo

${ }^{2}$ DIUMCE ED 2004-2005 de la Universidad Metropolitana de Ciencias de la Educación 
y Cabrera, 2005) han pretendido contribuir a la valoración y, desde la lingüística, al conocimiento de la estructura de esta lengua. En la Comunidad Sorda esta validación de su lengua ha promovido un sentimiento de orgullo por su cultura y su identidad sorda.

A partir de la década de los 90 se han promovido y promulgado sugerencias, normas y leyes tendientes a suscitar y motivar acciones que faciliten la integración de las personas consideradas con discapacidad, en los distintos ámbitos de la sociedad. Actualmente, la Ley 20.422, promulgada en Chile el 2010, que Establece Normas de Igualdad de Oportunidades e Inclusión Social de Personas con Discapacidad, en el Título IV, artículo 26 se señala: "Se reconoce la lengua de señas como medio de comunicación natural de la comunidad sorda”.

\subsection{Planteamiento del problema y objetivos}

En virtud de las leyes y normas promulgadas se han establecido los derechos de las personas sordas, tanto en la educación como en su integración a la sociedad. Asimismo, es importante destacar el reconocimiento que se ha hecho de la lengua de señas de las personas que conforman la Comunidad Sorda de Chile. A partir de esto, ha surgido la necesidad de disponer de un diccionario, que contribuya principalmente a la valoración de la LSCh y a facilitar su divulgación y aprendizaje. Se considera éste, también, como un material útil para ser consultado tanto por profesores, familias de niños y jóvenes sordos, como por toda persona interesada en este tema.

Por lo tanto, se ha planteado en este trabajo, como objetivo principal, la elaboración de un diccionario bilingüe que presente las señas (signos lingüísticos) de la LSCh, lengua de origen, y su traducción equivalente a las palabras del español, lengua meta.

La elaboración de este diccionario se constituye y se basa en un estudio lexicográfico, que ha implicado un proceso de indagación, selección, análisis, aclaraciones, decisiones y verificación de los datos relevantes, para fundamentar el registro de la LSCh. La necesidad de contar con un registro de señas de esta lengua es básicamente por el hecho de que es ocupada sólo en la comunicación cara a cara y por carecer de un sistema práctico de escritura que permita su difusión y conocimiento.

Es necesario señalar que la Comunidad Sorda de Chile frecuentemente ha solicitado la elaboración de diccionarios que registren sus señas. Por lo que en cumplimiento con estas demandas es que se elaboró el Diccionario Bilingüe LSCh/

(UMCE) “Una aproximación a la visión de mundo de las personas sordas a través del análisis de la Lengua de Señas Chilena". 
Español, que se ha sustentado en las teorías de la lexicografía que guían el estudio del campo léxico de una lengua.

\section{MARCO TEÓRICO}

\subsection{Lexicografía}

La lexicografía, como rama de la lingüística, permite dar fundamento a la tarea de construcción de diccionarios. Comprende un importante cuerpo de estudios teóricos que se relacionan con aspectos tales como la historia de los diccionarios, su estructura, su tipología, su finalidad y su relación con otras disciplinas afines, así como también la metodología para su elaboración y el análisis crítico de los diccionarios.

En general, los registros lexicográficos se preocupan de recopilar el léxico de un sistema lingüístico colectivo y de presentarlo por medio de un método descriptivo que idealiza el sistema. Se entiende por sistema colectivo, la lengua de una comunidad o minoría lingüística que permite la intercomunicación de sus miembros sin dificultad (Haensch, Wolf, Ettinger y Werner, 1982).

Actualmente, se emplean denominaciones como teoría lexicográfica, lexicografía teórica o metalexicografía para el componente teórico de la lexicografía, y diferenciarlo tanto de la práctica de la confección de diccionarios, como del ámbito disciplinario correspondiente. Ambos están diferenciados por sus objetivos y métodos de la lexicografía. De este modo, se distingue una lexicografía teórica de una lexicografía práctica. Pero, algunos autores como Hernández (en Medina, 2003) sugieren que se considere una definición de lexicografía que abarque el carácter tanto teórico como práctico de esta disciplina y, al mismo tiempo, que se contemple como un dominio más de la lingüística aplicada. Entonces, Hernández propone la siguiente definición: "La lexicografía es la disciplina de la lingüística aplicada que se encarga de los problemas teóricos prácticos que plantea la elaboración de diccionarios" (8).

Por otra parte, según Medina (2003), la metalexicografía estaría abarcando, como tema propio, tanto los aspectos teórico-metodológicos, que orientan la práctica o confección de diccionarios, como el diccionario concebido como objeto de estudio. La existencia de ese marco teórico ha hecho posible el cambio del tradicional desempeño del lexicógrafo a la disciplina lingüística (aplicada), dejando así de ser solo la técnica o el arte de componer diccionarios.

Los diccionarios, además de describir los significados de las palabras, tienen funciones sociolingüísticas que logran más que eso. La descripción de los significados junto con su pronunciación tiene indudablemente un contexto social. Los 
usuarios reciben, a través de los diccionarios, una forma del modelo estándar de su lengua, y esto constituye el punto de partida para reconocer las variantes que son posibles. Del mismo modo, se puede decir que los diccionarios también funcionan para reforzar y confirmar el estatus del sistema lingüístico de una lengua como tal. Los usuarios de una lengua minoritaria -como lo es la LSCh- por lo general, tienden a asumir que lo que usan para comunicarse es realmente una lengua cuando sus palabras se han registrado en un diccionario y, además, consideran que sólo las que aparecen en ese libro son las que pertenecen a esa lengua (Lucas, 2003).

\subsection{Diccionarios de lenguas de señas}

En general, los grupos de investigación sobre las lenguas de señas se han centrado en descripciones de los distintos aspectos de éstas como sistemas lingüísticos, para validarlas y aportar argumentos científicos para su reconocimiento oficial como lengua natural de las personas sordas. Asimismo, estos estudios han pretendido contribuir a su reconocimiento como el valor más importante de su cultura y símbolo de su identidad y, en el ámbito educativo, al derecho a ser educados en su propia lengua (Adamo, 2007).

Por otra parte, estos estudios también se han abocado a la elaboración de diccionarios, manuales y glosarios de lenguas de señas, como parte del trabajo de investigación lingüística. El primero de éstos, basado en 'principios lingüísticos', es el Diccionario de Lengua de Señas Americana, de Stokoe, Casterline y Croneberg (1960). Posteriormente aparecen otros, también basados en estos principios, como son los diccionarios de lengua de señas de Kenya, Italia, Gran Bretaña, Australia, Argentina, Uruguay y Colombia, entre otros, consignados por Massone y Druetta (2010).

En la realización de un diccionario de lengua de señas, como de cualquier otra lengua, el lexicógrafo ha de tomar como punto de partida la lengua misma, seleccionar los lexemas pertenecientes a ésta y presentarlos con su apropiada información lingüística.

Las lenguas de señas han de ser analizadas, pues, desde la lengua misma, en interacciones sordo/sordo y teniendo pleno conocimiento de la cultura sorda. El lingüista es un etnógrafo. Por lo que esta investigación puede ubicarse dentro de esta corriente de pensamiento lingüístico, que considera que la utilidad de un diccionario de lengua de señas tanto para el nativo de la lengua (sordos o hijos oyentes de padres sordos), como para los padres de niños sordos o bien para los estudiantes de lengua de señas, así como para todos aquellos especialistas que deseen manejarlo como obra de consulta, se basa en el hecho fundamental de que para comunicar a través de las 
señas se deben conocer sus significados, sus usos y la gramática y el discurso de la lengua a la que estas señas pertenecen (Massone y Druetta, 2010: 139-140).

Aquellos manuales, recopilaciones o inventarios de señas que presentan fotos o dibujos de las señas y la descripción de cómo éstas se realizan no se consideran diccionarios.

La mayoría de los diccionarios de señas son bilingües unidireccionales que van de la lengua de origen, la lengua de señas, a la lengua meta, la lengua oral. Se prioriza una de las lenguas y presentan un listado de señas con las glosas (nombre de la seña en imprenta mayúscula) que las identifican.

En relación a los diccionarios bilingües es necesario señalar la importante función que éstos pueden desempeñar. Por lo general se piensa que éste presenta sólo equivalentes de traducción en lugar de la definición semántica. De este modo, puede parecer que estos diccionarios se utilizan casi exclusivamente como instrumentos de traducción. Desde este punto de vista, se presenta una gran diferencia entre la descripción lexicográfica del diccionario semasiológico monolingüe y la del diccionario de traducción bilingüe. En el primero se describen los contenidos que pueden corresponder a determinados significantes de una lengua y, en el segundo, los significantes de una lengua de destino que pueden corresponder a determinados significantes de una lengua de origen. En realidad, los diccionarios bilingües, además de ser usados como instrumentos para la traducción, se emplean también, y con frecuencia, del mismo modo que los diccionarios semasiológicos y onomasiológicos (Haensch et al., 1982).

En los diccionarios bilingües, para la representación del lema generalmente se utiliza un dibujo o una fotografía de la seña y muy pocas veces se emplea en su transcripción un sistema de notación que dé cuenta de sus componentes formacionales; éste es usado exclusivamente por investigadores para el análisis de las unidades de estas lenguas. La mayoría de estos diccionarios presentan distintos modo de ordenar las entradas. Por lo general, agrupan las señas en áreas de contenidos y dentro de cada área las glosas se ordenan alfabéticamente (Riekehof, en Johnston, 2003) y a veces se agrupan señas según el parámetro formacional que sobresale. Éste puede ser la configuración manual, la locación o la dirección del movimiento de la seña que se clasifica.

Los diccionarios bilingües bidireccionales tienen una sección que va de la lengua de señas a la lengua mayoritaria y también ofrecen a los usuarios la opción de seleccionar una palabra de la segunda lengua (hablada y escrita) y luego encontrar su equivalente en la lengua de señas. Esto se puede hacer de una manera simple, a través de un índice o por medio de una segunda sección del diccionario en que las palabras claves, ordenadas alfabéticamente, guíen directamente hacia los signos equivalentes y sus traducciones, como un diccionario de señas bilingüe y unidireccional (Johnston, 2003). 


\subsection{Lengua de señas}

La lengua de señas se trata de una lengua de naturaleza gestual-visual. Se realiza mediante las manos que se mueven en el espacio o en contacto con el cuerpo, acompañadas de movimientos corporales y expresiones faciales por parte de las personas que se expresan y los mensajes se reciben por medio del sentido de la visión. Corresponde a un sistema lingüístico con una gramática particular, puesto que organiza sus signos de acuerdo con reglas que definen los distintos niveles de su estructura (Acuña, Adamo y Cabrera, 2009).

Gran parte del conocimiento que se tiene de la LSCh es el resultado de varias investigaciones llevadas a cabo por el equipo de estudio que conforman las autoras de este artículo. Durante los años 1991 y 1992 también se realiza un trabajo de análisis semántico y recopilación de señas de la Comunidad Sorda de Concepción (Pilleux, Ávalos y Cuevas, 1991, 1992), en la Universidad Austral, que no tuvo mayor continuidad hasta hoy.

Los parámetros formacionales que conforman las unidades léxicas de las lenguas de señas se clasifican principalmente en cuatro clases. Cada una de estas clases contiene una lista finita de unidades que se consideran equivalentes a los fonemas de las lenguas orales, puesto que funcionan como unidades distintivas de los signos mínimos; para la descripción de algunas señas es necesario agregar la clase 'rasgos no manuales', como un constituyente más que funciona también como unidad distintiva (Adamo, 1994).

Los constituyentes de las señas son:

- Configuración de las manos (CM). Formas que una o las dos manos adoptan en la realización de las señas.

- Movimiento (M). El que se ejecuta durante la realización de las señas.

- Lugar (L). Donde se realiza la seña, en el espacio o en partes del cuerpo del señante.

- Orientación (O). La orientación que adoptan las palmas de las manos durante la realización de la seña; hacia arriba, abajo, al frente o a los lados.

- Rasgos No Manuales (RNM). Corresponde a expresiones o movimientos faciales y del cuerpo que ocurren en la realización de la seña.

Entre las características que más se destacan en la lengua de señas son el carácter visual de sus unidades lingüísticas y el uso del espacio. Este último, que forma 
parte del sistema, corresponde al espacio que se encuentra frente o en el entorno más próximo del señante, y es sumamente explotado en la descripción de eventos. Esta característica hace que éste sea un sistema complejo que brinda mucha información de manera polisintética haciendo uso del espacio tridimensional.

Otro elemento importante dentro del sistema de la lengua de señas corresponde a los 'clasificadores', unidades complejas cuya función es la de hacer una referencia anafórica en el discurso, por medio de una seña en la que sus componentes, específicamente configuración de la mano y movimiento, representan cada uno simultáneamente una significación distinta (Adamo et al., 2003). Dadas estas características formales y semánticas, presentan un desafío para su traducción, debido a que no se cuenta con una expresión equivalente correspondiente al español. Además, según los contextos, su significación varía, por lo que esto dificulta su registro en un diccionario.

\subsection{La categoría verbo en la LSCh}

Un aspecto interesante que surge en el estudio del sistema lingüístico de la LSCh es el de la categoría gramatical verbo. Es importante abordarlo aquí ya que es la categoría que en el diccionario aparece con su correspondiente clasificación.

En los estudios para el análisis de los verbos de la LSCh (Adamo et al., 1999 y Acuña et al., 1999) se adoptó la clasificación propuesta por Padden $(1983,1990)$ y otros como Liddell (1990) y Lucas y Valli (1990). Según estos autores se establece una clasificación que distingue tres categorías:

a) Verbos Plenos: Son verbos no deícticos que entregan la información semántica correspondiente, más aspecto y/o manera. Todos estos verbos necesitan marcar, con otra seña, los roles gramaticales.

b) Verbos de Concordancia: Son verbos deícticos que cambian la direccionalidad del movimiento según los roles gramaticales de sus argumentos en la oración, es decir, sujeto y objeto. Desde el punto de vista formal, las modificaciones que ocurren en estos verbos son consecuencia de cambios en el parámetro de Movimiento y su direccionalidad, para aportar la información de quién es la persona que realiza o recibe la acción.

c) Verbos Espaciales Locativos: Esta categoría incluye verbos deícticos que expresan, por medio del parámetro Movimiento, la locación del punto inicial y final de la acción señalada, como también una locación específica en la cual ocurre una acción en un contexto determinado. 
En los verbos espaciales, el Movimiento formal de la seña no tiene como función, como en los de concordancia, marcar los roles semánticos entre los argumentos, sino que corresponde a una representación del movimiento (real o a escala) de entidades relacionadas con el verbo. De esta manera, el Movimiento cumple una doble función: ser un parámetro formacional y especificar movimiento y localización de entidades del predicado.

Aun cuando todavía esta última clasificación sigue vigente, está siendo revisada desde una dimensión cognitivo-funcionalista que intenta explicar la descripción de las lenguas de señas desde puntos de vista distintos, especialmente orientada a observar las peculiaridades que ofrece la modalidad viso-espacial de estas lenguas (Morales-López, Boldú, Alonso, Gras y Rodríguez, 2005).

Según Engberg-Pedersen (1993, en Morales-López et al., 2005):

The 'verbal' meaning not only depends on the movement units, but on the interaction of de classificatory morpheme (expressed by the handshape) and the morphemes expressed by the movement. Or put differently, the choice of handshape unit contributes to the productive meaning of the verb complex, not just the classificatory meaning [el significado 'verbal' no sólo depende de las unidades de movimiento, sino de la interacción del morfema clasificatorio (expresado por la configuración manual) y los morfemas expresados a través del movimiento. $\mathrm{O}$ dicho en otros términos, la elección de una configuración manual determinada contribuye al significado del predicado del verbo complejo, y no solamente al significado clasificatorio] (245).

De este modo, se quiere indicar que el significando de estos verbos, y por lo tanto del predicado, depende de la Configuración de las Manos en interacción con los morfemas de Movimiento.

En consecuencia, aceptando esta interacción de los morfemas componentes, se habla así de raíces verbales y no de clasificadores, ya que las configuraciones manuales no sólo tienen la función de clasificar a los referentes representando sus características que más se destacan, sino que además tienen otras funciones relacionadas con la manera cómo se presenta el evento que se describe (caminar con dificultad, si se choca con otra persona o si sale sigilosamente de un lugar determinado). Engberg-Pedersen (en Morales-López et al. 2005) denomina a los verbos de esta clasificación, por sus características enunciadas, verbos polimorfémicos.

\section{METODOLOGÍA}

La investigación lingüística, como señalan Massone y Druetta (2010), no es neutra y en su desarrollo, como en toda ciencia, se construyen relaciones de poder y 
los lingüistas deben controlar este poder para evitar ejercer violencia epistémica sobre el objeto de estudio, como son las lenguas de comunidades sordas a las que no se pertenece. De este modo, los autores sugieren que:

El lingüista debe convertirse en etnógrafo y construir el conocimiento del otro junto con el otro, e interpretar la lengua en contexto, más aún cuando se trata de una lengua en contacto y en contexto, dado que solo es conversacional, es decir, de práctica interpersonal y se encuentra en permanente contacto con el español de nuestros países latinoamericanos (2010: 149).

Para la elaboración del Diccionario Bilingüe Lengua de Señas Chilena/Español se requirió de un equipo en el que participaron personas sordas, nativas en lengua de señas (personas sordas de familias sordas) y otros miembros de la Comunidad Sorda. El rol de estas personas en la construcción de este diccionario ha consistido en un proceso de indagación, de interpretación, de elaboración y verificación, y validación de los datos relevantes necesarios. Su participación activa fue aportar con sus conocimientos de usuarios nativos de su lengua.

En general, la elaboración de diccionarios implica una investigación lexicográfica que presenta gran complejidad ya que es necesario tener en cuenta, en su preparación, distintos aspectos. En el caso de una tarea lexicográfica dedicada a la elaboración de un diccionario de lengua de señas, interesa discutir todos los aspectos relacionados con este proceso, tales como: la recolección del material lingüístico; la selección de las unidades léxicas; quiénes las informan; las diferentes posibilidades con que se cuenta para la organización del material recolectado; la validación del material lingüístico, y todo ello, sobre la base de las diferentes finalidades que se consideran para la utilidad de un diccionario.

Frente a estas observaciones, una de las principales actividades en este trabajo se centra en la selección de las señas que fueron incluidas en el Diccionario Bilingüe LSCh/Español (Acuña, Adamo y Cabrera, 2009). Por un lado, un aspecto importante corresponde a determinar cuáles señas serán tratadas como unidades léxicas, lo que representa un primer problema como desafío lexicográfico. Este es un estudio específico, muy significativo, porque de él dependen qué señas se registrarán como entradas léxicas. Según Johnston y Schembri (en Johnston, 2003), un criterio que puede ser de utilidad para determinar una unidad léxica es que exista una unidad cuya forma de realización sea claramente identificable, y que se pueda asociar en forma consistente y sostenida a un significado dado, aunque la unidad se produzca fuera de contexto, lo que se conoce comúnmente como su 'forma de citación'.

Esta distinción del carácter léxico de las señas ha sido necesaria porque existen señas que no tienen una significación estable, motivado esto por su función deíctica, ya que según la manera y dirección de los movimientos, como su significación, 
variarán de acuerdo con las innumerables posibilidades que puede ofrecer el uso del espacio y el contexto situacional y lingüístico (Adamo et al., 2003).

Por otro lado, como segundo problema y desafío lexicográfico, se debió confirmar que las señas seleccionadas fueran las que estuvieran actualmente en uso por la Comunidad Sorda, condición que, en ocasiones, significó dejar fuera de esta recopilación un conjunto de señas que son utilizadas por grupos comunitarios muy reducidos, especialmente en ámbitos cerrados como podría ser una escuela especial. El tener en cuenta estas realidades valida las unidades consignadas.

Asimismo, un tercer desafío ha consistido en tomar decisiones en cuanto a la forma de representación de las señas. Esto último, de considerable importancia, se refiere a la necesidad de encontrar la mejor manera para registrar e ilustrar, en un formato bidimensional, como lo es el papel, el sistema léxico de una lengua que es fundamentalmente de naturaleza gestual-visual y que se realiza en la triple dimensión del espacio más la dimensión temporal. Estos aspectos han sido analizados, discutidos y aclarados para tomar decisiones sobre el registro de las unidades léxicas.

Por tratarse este trabajo de la elaboración de un diccionario de carácter bilingüe, se han considerado tanto la lengua de origen, la lengua de señas chilena, como la lengua de destino o meta, el español. Para la recolección de las unidades léxicas de la LSCh, las personas sordas, en conjunto con el equipo, elaboraron listas de señas relacionadas con campos semánticos, para facilitar la elicitación del léxico de parte de quienes aportaron las señas.

En cuanto a la selección de este léxico, a partir de un criterio semasiológico, se decidió consignar las categorías de uso más general y cotidiano. Las señas, realizadas por las personas sordas participantes, se registraron en fotografías digitales. En este proceso se consideraron aspectos técnicos tales como fondo, iluminación, ángulo de la toma y también normas para la vestimenta y accesorios, entre otros. Con el cuidado de estos detalles, en las sesiones de fotografías y, posteriormente, en el procesamiento de limpieza de las fotos, se contribuyó a una presentación de las señas ordenada, inteligible y sin ambigüedad.

El siguiente paso consistió en agregar a las imágenes una simbología, preparada especialmente para representar de la manera más fiel posible los movimientos y cambios originados en la producción de las señas. Con esto se dio por finalizada la preparación de las unidades léxicas para su presentación en el diccionario.

\subsection{Macroestructura}

La macroestructura del diccionario la constituyen distintas partes relevantes que son: una introducción que explica la finalidad, a quiénes está dirigido, cómo se obtuvo el material lingüístico, entre otras; las instrucciones de uso, las abreviatu- 
ras y la simbología usada para la representación de cómo se realizan las señas; y el cuerpo del diccionario que comprende el material lingüístico recopilado. La parte más relevante de la macroestructura la constituye el conjunto de entradas léxicas dispuestas de acuerdo a un criterio ordenador.

Una vez seleccionado el léxico y procesadas las fotos digitales se procedió a elaborar el diseño de los artículos. Las unidades léxicas se presentaron, pues, en una macroestructura, es decir, en una sucesión de artículos independientes o entradas. Este diccionario, de carácter semasiológico, sigue el principio 'del significante al significado'. Se decidió, para la ordenación de las unidades, el orden alfabético, ya que tiene como ventaja la rapidez y la simplicidad cuando se quiere buscar una entrada, pero, como dice Kocjančič (2004), no se consideran las relaciones semánticas que existen dentro del vocabulario.

\subsection{Microestructura}

La microestructura de los diccionarios se constituye en el contenido y la organización de un artículo lexicográfico. En el artículo se presenta la unidad mínima autónoma en que se organiza el diccionario (Garriga Escribano, en Farkačová, 2010). Se trata, por lo tanto, de la base del diccionario donde se dan informaciones acerca de la palabra o la unidad léxica correspondiente.

En este diccionario bilingüe, cada artículo se compone de dos partes fundamentales: la enunciativa y la informativa o definitoria. Y, como aclara Porto Dapena (2002), la entrada léxica se caracteriza por tener carácter abstracto y formar parte de la macroestructura del diccionario, mientras que el lema y el enunciado son formas concretas de la palabra-entrada y forman parte del artículo lexicográfico o microestructura del diccionario. La macroestructura, señala Farkačová (2010), representa una lectura vertical del diccionario mientras que la microestructura constituye una lectura horizontal.

Es así que en la estructura de los artículos se muestra el lema que corresponde a una seña por medio de una fotografía, o más de una, las que pueden aparecer montadas o una al lado de la otra, según convenga por la imagen; esto sucede cuando la realización de la seña presenta cambios de formas de las manos o tiene varios movimientos.

La foto se complementa con información gráfica, a través de una simbología elaborada específicamente, la cual permite interpretar el movimiento que corresponde a cada seña. A la derecha de la foto se presenta una glosa, es decir, una palabra del español escrita en imprenta mayúscula que representa el concepto de la seña. Estas entradas léxicas están organizadas, como ya se ha dicho, en orden alfabético, como una manera de facilitar al usuario su ubicación en el texto. Debajo 
de la glosa se registra, en el caso que corresponda a un verbo, la clasificación que éste tiene en el sistema de la lengua de señas chilena (Adamo et al., 1999, Acuña et al., 1999).

En la segunda parte del artículo se dan varias informaciones sobre el enunciado, una de ellas es la definición que sigue a la glosa. Ésta se elaboró según el significado que la seña tiene para la Comunidad Sorda, a partir de la información semántica aportada por sus miembros y, en algunos casos, fue necesario aclarar ésta con el Diccionario de la RAE (1992). Según los propósitos definidos y los destinatarios hacia quienes está dirigida esta obra, se ha tenido especial cuidado de contar con definiciones que, aunque son informativas no suelen ser muy extensas, lo que facilita la comprensión de esta información a los usuarios sordos.

En el cuerpo del artículo también se consideró la lengua de destino, el español. En primer lugar se registra la categoría gramatical correspondiente y la traducción de la seña con la unidad léxica específica del español, con sus posibles sinónimos y antónimos de uso más común, cuando corresponde.

Ejemplos de artículos del diccionario bilingüe LSCh/Español:

\section{AMASAR}

Verbo pleno

Sobar la masa preparada con harina, agua o leche y otros ingredientes como aceite, manteca o margarina.

Esp.: v. tr. Amasar, sobar.

\section{BANDERA}

Pedazo de tela, generalmente rectangular, sujeto a un palo o mástil que, según su color o dibujo, constituye el símbolo de una nación u otra colectividad. Esp.: sust. f. Bandera, pabellón, estandarte, insignia.

En las últimas etapas de este trabajo se han llevado a cabo diversas acciones para evaluar y validar los distintos aspectos de este diccionario. En primer lugar, para la evaluación participaron personas sordas que revisaron cada una de las fotografías, quienes contribuyeron con sugerencias para la representación de los movimientos. Por otra parte, con ellas se revisó la definición, lo que permitió asegurar que la acepción allí redactada correspondiera efectivamente a aquella que se asigna en la LSCh por la Comunidad Sorda.

Finalmente, como último paso, fue solicitada una revisión del material por parte de un grupo de miembros de la Comunidad Sorda que no había participado en ninguna otra etapa del proceso, quienes cumplieron con la función de jueces evaluadores, para confirmar, de esta forma, que las señas consignadas pertenecieran a la lengua de esta comunidad. 


\section{DISCUSIÓN}

En este apartado se discute acerca de los tres problemas planteados como desafíos lexicográficos que se presentan en la elaboración de un diccionario de lengua de señas y específicamente en el Diccionario Bilingüe Lengua de Señas Chilena/Español.

En relación al primer problema, se destaca la necesidad de distinguir entre señas reconocidas como léxicas de otras que no lo son, y tomar decisiones con respecto a su registro. Las señas consideradas léxicas presentan características específicas, como ser perfectamente identificables y mantener un significado relativamente estable en los distintos contextos en que se presenten. El factor visual-gestual que caracteriza a esta lengua influye en el sistema, de modo tal que existen señas que no cumplen con los requisitos de lexicalidad. Son unidades que tienen una marcada función deíctica, como son aquellas señas que indican a las personas que intervienen en el diálogo, equivalentes a los pronombres personales.

Estas señas están constituidas con la misma configuración manual, el dedo índice como indicador, y lo que cambia es la direccionalidad del movimiento, el que no tiene una dirección estable porque depende de la localización de los referentes en el contexto situacional. De este modo, por la variabilidad de su direccionalidad, no es posible reconocer su significación fuera de contexto, incluso para un usuario, excepto la de aquella que indica 'el que habla'.

Entonces, se puede decir que estas señas deícticas representan sólo las nociones significativas de 'YO' opuesta a 'NO-YO'. La primera se indica con el índice señalando al señante y en el segundo caso, el índice señala hacia el espacio frente a él o en torno a él, para representar las otras personas gramaticales. Y esto, sin tener un punto espacial determinado para cada una de ellas (Cabrera, 1995). Las características de gestos como éstos, propias de las señas netamente deícticas, otorgan una ambigüedad que está dada por apuntar al referente o el lugar que éste ocupa, lo que variará según el contexto de la situación comunicativa. Por esta razón no fueron incluidas en el diccionario.

También existen señas que, aun cuando son unidades léxicas, quedaron fuera de la selección por tratarse de señas que se usan para indicar las partes 'visibles' del cuerpo. Todas ellas se realizan con una misma configuración manual: la mano empuñada y el dedo índice extendido y se mueven naturalmente, para indicar la parte del cuerpo de la cual se habla, por lo cual, lo único que cambia es el parámetro Lugar. Dada la transparencia en el significado de cada una de estas señas, no fueron registradas en el diccionario.

Otro grupo que fue descartado correspondió a señas deícticas que se usan para indicar relaciones espaciales, tales como ARRIBA y ABAJO, que se realizan por medio de la indicación hacia esas direcciones. Dada las infinitas posibilidades direccionales hacia las cuales se puede señalar (bajo la mesa, en el subsuelo, arriba de 
la cama, en el segundo piso) no es posible rescatar el carácter discreto de unidades espaciales como éstas.

El segundo problema y desafío lexicográfico que se presentó en la selección de las unidades ha sido el requisito de que éstas fueran señas reconocidas por un grupo importante de la comunidad sorda. Para confirmar esto se incorporaron activamente personas sordas, hijas de familias sordas, quienes validaron la inclusión de estas señas. Se cauteló que entre las personas, que participaron en el registro, hubiera diferencia de género así como diferencia etaria, de manera tal de validar la vigencia de dichas señas en diversos ámbitos socio-comunicativos.

El tercer problema se planteó con la representación de las unidades léxicas. La naturaleza gestual-visual de la lengua de señas ha sido siempre un desafío para todos los que elaboran diccionarios o manuales. Esto es porque se pretende fijar en el papel, a través de dibujo o fotografía, una unidad léxica que su realización es por medio del movimiento. Los avances tecnológicos permitieron que se monitoreara la toma de las fotografías digitales durante el proceso para poder seleccionar su mejor ángulo y, de este modo, obtener una clara imagen de la seña.

Además, en esta tarea, fue necesario tener en cuenta que la fotografía así tomada facilitara la labor posterior de añadir la simbología para mostrar los movimientos que requiere la seña. Por lo tanto, el siguiente paso consistió en el procesamiento digital de cada una de las fotografías, interviniéndolas para llegar a tener una imagen óptima en tamaño y calidad, que reflejara claramente la realización de la seña.

Para la elaboración de otros diccionarios de lengua de señas se sugiere que la definición del significado se recoja por medio de grabaciones donde una persona sorda le dé la explicación a otra en lengua de señas. Luego, la grabación se transcribirá y traducirá para la definición del artículo. Otra posibilidad de tener en cuenta, consiste en que el diccionario bilingüe sea bidireccional, para facilitar así la búsqueda de las unidades que se quiere conocer.

\section{CONCLUSIONES}

El trabajo lexicográfico para un diccionario bilingüe de lengua de señas y lengua oral se trata de un estudio complejo, en el que es necesario conciliar tareas que han de realizarse entre lexicógrafos y personas sordas competentes en su lengua. La situación ideal es la formación de equipos de trabajo en el que participen personas sordas como lingüistas, tanto para la elaboración de diccionarios como para los estudios sobre el sistema y uso de la lengua de señas. Esto ha sido posible en países donde las personas sordas, interesadas en el tema, han tenido la posibilidad de realizar estudios superiores.

En la elaboración de este Diccionario Bilingüe: LSCh/Español, se estableció 
la distinción entre las unidades léxicas y las que no lo son, basada en el comportamiento formal y semántico de las señas. Esto permitió organizar la selección del léxico, considerando conjuntamente los aportes de los informantes sordos.

La participación de las personas sordas ha sido uno de los aspectos más relevantes en este trabajo, por la validación necesaria que sólo puede estar dada por usuarios nativos de esta lengua, quienes fundamentalmente proceden de familias de sordos. Por lo tanto, el léxico que contiene este diccionario ha sido entregado, confirmado y validado por miembros de la Comunidad Sorda, quienes han participado activamente en todas las etapas de su elaboración, dándole así un fundamento de legitimidad.

El diseño y la organización de los artículos, la parte más importante de un diccionario, provee la información específica en forma unidireccional sobre el léxico de la LSCh, lengua de origen, su definición y la clasificación de sus verbos, y la traducción al español, lengua meta, con sus sinónimos y antónimos cuando correspondía.

Dado que este Diccionario corresponde a una versión en papel, fue definida la representación de sus unidades léxicas a través de fotografías digitales, procesadas según los requerimientos de una imagen suficientemente clara y con indicaciones de sus movimientos con una simbología específica.

Contar con un registro lexicográfico de una lengua es algo que permite que la misma comunidad usuaria pueda dar valor al sistema que ocupa en forma cotidiana para la comunicación, más aún, cuando se trata de una lengua que pertenece a una comunidad minoritaria, que carece de escritura, y sólo se transmite en la comunicación cara a cara. Así, este Diccionario Bilingüe pretende ser una obra instrumental para difundir la lengua, pero también, y con una relevancia mayor, ser un valor patrimonial para esta comunidad lingüística.

Si bien es cierto que un diccionario de estas características y con la finalidad que tiene es deseable en primera instancia que sea presentado en soporte de papel, es también imperiosa la necesidad de una versión de características tecnológicas que den la posibilidad de conocer visualmente la realización de las señas. Estos diccionarios en formato digital permiten dar cuenta de manera más natural la verdadera naturaleza visual de sus signos.

\section{REFERENCIAS}

Acuña, Ximena. (1997). Estudio Descriptivo del verbo de concordancia en la LSCh. Tesis inédita de maestría. Pontificia Universidad Católica de Chile, Santiago, Chile.

Acuña, X., Adamo, D. y Cabrera, I. (2005). Narraciones en la lengua de señas, 
una aproximación a la cultura sorda. II Congreso Nacional de la Lengua de Signos Española. Valladolid. España.

Acuña, X., Adamo, D., y Cabrera, I. (2009). Diccionario bilingüe Lengua de Señas Chilena/Español. Tomos I y II. Santiago: MINEDUC, UMCE.

Acuña, Ximena, Adamo, Dora, Cabrera, Irene, y Lattapiat, Pamela. (1999). Verbos espaciales locativos en la lengua de señas chilena. RLA, 37, 7-21.

Acuña, X., Adamo, D., Cabrera, I. y Lissi, M. R. (2012). Estudio descriptivo del desarrollo de la competencia narrativa en la lengua de señas chilena. Onomazein, 2, (26), 193-219.

Adamo, D. (1994). Estudio descriptivo de los parámetros básicos de la estructura sublexical de la lengua de señas chilena. (Tesis inédita de maestría). Pontificia Universidad Católica de Chile.

Adamo, D. (2007). Lexicografía y diccionarios de lenguas de señas. En S. Cvejanov (ed.), Lenguas de señas. Estudios de lingüistica teórica y aplicada (pp.173184). Neuquén, Argentina: Editorial de la Universidad del Comahue.

Adamo, D. y Cabrera, I. (1989). La Comunidad de Sordos: Una minoría lingüística. Octavo Seminario Nacional de Investigación y Enseñanza de la Lingüística. Sociedad Chilena de Lingüística. Universidad de Santiago de Chile, Santiago, Chile.

Adamo, D., Cabrera, I. Lattapiat, P. y Acuña, X. (1999). Verbo de concordancia en la lengua de señas chilena. Onomazein, 4, 335-344.

Adamo, D., Acuña, X., Cabrera, I. y Lattapiat, P. (2003). Clasificadores en la Lengua de Señas Chilena. En M. Simón, V. Buscaglia y M. I. Massone (eds.), Educación de sordos: ¿Educación especial y/o educación? (pp. 82-93). Editorial LibrosEnRed [en línea]. Disponible en www.librosenred.com.

Barrientos, C. y Tenorio, V. (1987). Manual de lenguaje de señas. Temuco IX Región (Chile). Santiago: Editorial Interamericana.

Cabrera, Irene. (1995). Estudio descriptivo de los pronombres personales en la lengua de señas chilena. Tesis inédita de maestría. Pontificia Universidad Católica de Chile.

Farkačová, Tat'ána. (2010). La microestructura de los diccionarios bilingües español-checos. Magisterská diplomová práce. Universitas Masarykiana Bbrunensis. Facultas Philosophicas [en línea]. Disponible en http://is.muni.cz/ th/145624/ff_m/diplomova_prace.pdf. Consulta: 23.07.2013.

Haensch, Günther, Wolf, Lothar, Ettinger, Stefan y Werner, Reinhold. (1982). La lexicografia: De la lingüistica teórica a la lexicografía práctica. Madrid: Gredos.

Johnston, Trevor. (2003). Language standardization and signed language dictionaries. Sign Language Studies, 3 (4), 431-468.

Kocjančič, Polonca. (2004). Acerca de la macroestructura y la microestructura en el diccionario bilingüe. Verba hispánica, 12, 171-185. 
Lattapiat, Pamela y Marín, Juan. (2000). Manual de lengua de señas. Santiago: Universidad Metropolitana de Ciencias de la Educación. Impreso en Talleres UMCE.

León, Gloria, Merino, René y Villarroel, Rodolfo. (1978). Inventario Preliminar de Gestos Utilizados por Niños con Trastornos Auditivos. Seminario de Título Especialista en Educación de Sordos. Universidad de Chile.

Liddell, Scott. (1990). Four functions of a locus: Reexamining the structure of space in ASL. En Ceil Lucas (ed.), Sign language research: Theoretical issue. Washington DC: Gallaudet University Press.

Lucas, Ceil. (2003). The role of variation in lexicography. Sign Language Studies, 3 (3), 322-340.

Lucas, Ceil y Valli, Clayton. (1990). Predicates of perceived motion in ASL. En S. Fischer y P. Siple (eds.), Theoretical issues in sign language research (pp. 153166). Chicago: University of Chicago Press.

Massone, María Ignacia y Johnson, Robert. (1990). Kinship terms in Argentine sign language. Sign Language Studies, 73, 347-360.

Massone, María Ignacia y Druetta, Juan Carlos. (2010). Diccionarios de lengua de señas: Cuestiones lexicográficas. Revista de Investigación, 70 (34) 129-154.

Medina Guerra, Antonia María. (2003). (coord.). Lexicografía española. Barcelona: Ariel.

Morales-López, E., Boldú, R. M., Alonso, J. A., Gras, V., y Rodríguez, M. A. (2005). The verbal system of Catalan sign language (LSC). Sign Language Studies, 5, 4, 441-496.

Padden, Carol. (1983). Interaction of morphology and syntax in American sign language. Doctoral Dissertation. San Diego: University of California.

Padden, Carol. (1990). The relation between space and grammar in ASL verb morphology. En C. Lucas (ed.), Sign language research. Theoretical issues (pp. 118-132). Washington D.C.: Gallaudet University Press.

Pilleux, Mauricio, Ávalos, Erika y Cuevas, Hernán. (1991). Lenguaje de señas: Análisis sintáctico-semántico. Valdivia: Ediciones Universidad Austral de Chile.

Pilleux, Mauricio, Ávalos, Erika y Cuevas, Hernán. (1992). Diccionario del lenguaje de señas de Concepción. Valdivia: Ediciones Universidad Austral de Chile.

Porto Dapena, José-Álvaro. (2002). Manual de técnica lexicográfica. Madrid: Arco/ Libros.

Real Academia Española. (1992). Diccionario de la lengua española. Tomos I y II. Madrid: Espasa Calpe.

Stokoe, W. C., Casterline, D. C. y Croneberg, C. G. (1960). A diccionary of American sign language on linguistic principles. Washington, D.C.: Gallaudet College Press. 